\title{
Immunologische Untersuchungen der organspezifischen und zytotoxischen Eigenschaften des Antiserums der Leukozyten des Kaninchens.
}

\author{
Von \\ Dr. med. Hiroyuki Yamamoto. \\ (山朴业行) \\ (Aus der Gerichitlich-medizinischen Institut der Kaiserlichen Tohoku- \\ Universität. Direktor: Prof. Dr. med. Tetsuro Ishikawa.) \\ I. Über die Organspezifität der einzelnen \\ Leukozyten. \\ Dass das Hämoglobin (Klein, ${ }^{1)}$ Lerrs, ${ }^{2}$ Higashi, ${ }^{3,4)}$ Hektoen und \\ Schulhof ${ }^{5)}$ ), und die Lenkozyten (Salus, ${ }^{6)} \operatorname{Iida}^{7)}$ ), der Säugetiere Art- \\ und Organspezifität besitzen, ist eine wohlbekannte Tatsache. ILesch ke $\mathrm{e}^{8)}$ \\ berichtete zum erstenmal über die Artspezifität der Leukozyten, doch wur- \\ den seine Ergebnisse von Spät $\mathrm{t}^{93}$ bestritten. Es dürfte sich aber meines \\ Erachtens bei den Leukozyten nicht nur Organspezifitït, sondern auch \\ ziemlich strenge immunologische Differenz zwischen den einzelnen Leu- \\ kozytenarten zeigen. In vorliegender Mitteilung soll das Frgebnis meines \\ Experiments wiedergegeben werden.
}

\section{Antigen und Antiserum.}

Polynukleären Leukocyten von Kaninchen habe ich nach dem Verfahren von Spät als Antigen gesammelt, obschon es dafür noch ver-

1) Kle in, A., Wien. klin. Wochenschr., 1903, 16, 117.

2) Leers, O., Centralbl. f. Bakteriol. (I. Abt.), 1910, 54, 1.

3) Higashi, S., Journ. of Biochern. (K a kiu chi), 1983, 2, 315.

4) Higashi, S., Kokkaigakkai-Zasshi, 1921, 539.

5) Hektoen u. Schulhof, Journ. of Infect. Diseas. 1923, 33, 224.

6) Salus, G., Biochem. Zeitschr., 1914, 60, 1.

7) I ida, E., Tokio Igakukwai Zasshi, 1924, 38, 1121.

8) Leschke, E., Zeitschr. f. Immunitütsf., 1913, 16, 627.

9) Spät, W., Zeitschr. f. Immunitätsf., 1914, 21, 565. 
schiedene andere Methode gibt (Neumann, ${ }^{10} \mathrm{Fleming},{ }^{11)} \mathrm{Hirsch}^{12}$ ). Ungefähr $100 \mathrm{ccm}$ Rindfleischbouillon wurde cinem Kaninchen unter sterilen Kautelen danach die Höhle mit physiologischer Kochsalzlösung einige Male ausgespült und dann von dieser Spülfiüssigheit die bezüglichen Zellen durch Zentrifugieren getrennt. Nachden der gewonnene Zellbrei $(1,5-2,0 \mathrm{~g})$ zur Entfernung der beigemischten Bouillon mehrere Male mit physiologischer Kochsalzlösung gewaschen worden war, liess er in 5-10 ccm physiologischer Kochsalzlösung wieder gleichmüssig teilen. Diese Suspension wurde als Antigen in die Flügelvenen einer Hausente über zwanzig Male mit vier- oder fünftägigem Intervall injiziert. Das Verhältnis der in dieser Suspension vorhandenen Ienkozyten war, wie mikroskopisch nachgewiesen wurde, folgendes: Polynukleäre 89,\%, Mononukleäre $9,3 \%$, und Eosinophile $0,8 \%$. Wie man hicraus ersieht, besteht das Antigen hauptsächlich aus polynukleären Leukozyten, deshalb betrachte ich es als polynukleäres Leukozyten-Antigen und das ihm entsprechende Immunserum als polynukleäres Leukozyten-Antiserum oder Leukotoxin.

2. Über die Organspezifitä der Leukozyten, insbesondere der polynukleären.

Um zu entscheiden, ob das auf die oben geschilderte Weise dargestellte polynukleäre Leukozyten-Antiserum, wie crwartet, strenge Organspezifität besitzt, d. h. nur gegen polynukleäre Lcukozyten immunologisch reagiert oder nicht, suchte ich durch Prüzipitinreaktion mit den Wasserextrakten polynukleärer, mononukleürer und eosinophiler Leukozy ten und einiger anderer Organe die einzelnen Leukozytenarten zu identifizieren. Solche Wasserauszüge wurden mit physiologischer Kochsalzlösung aus den genannten, im Mörser zerstossenen Zellen und Geweben hergestellt und die brauchbare Oberschicht durch Zentrifugieren von dem unlöslichen Sediment getrennt. Die drei Arten der Leukozyten und der Zellbrei einiger Organe wurden anf folgende Weise dargestellt.

(a) Polynukleäre Leukozyten. Wie oben erwähnt, wurden sic nach dem Verfahren von Spät aus einem Kaninchen genommen. Es sei noch hinzugefügt, dass die in diesem Falle zur Leukozy tengewinnung verwendete Bouillon einerseits aus Rind-, anderseits aus Hundefleisch bereitet wurde,

10) Neumann, Alf., Wien. klin. Wochenschr., 1926, 39, 773.

11) Fle in ing, Al., Brit. Journ. of Exper. Pathol., 1926, 7, 281.

12) Hirsch, R., Pflügers Arch., 1927, 216, 506. 
um eine durch Präzipitinreaktion infolge des beigemischten Bouilloneiweisses mögliche Verwicklung des Versuchsresultats zu vermeiden.

(b) Mononukleäre Leukozyten. Zur reinen Darstellung dieser Leukozy ten wurde einem Kaninchen anstatt Bouillon nach dem Spätschen Verfahren $40 \mathrm{ccm}$ einer $0,003 \%$ igen wässerigen Pilokarpinlösung in die Peritonealhöhle eingeführt (Besrdkas Verfahren) und nach 24 Stunden die Höhle mit physiologischer Kochsalzlösung mehrmals ausgespült. Die Spülflüssigkeit wurde wie bei (1) weiter behandelt. Auf diese Weise kann man einen Leukozy tenbrei bekommen, der wie mikroskopisch nachgewiesen wurde, fast ausschliesslich aus Mononukleären besteht t $^{\prime}$

(c) Eosinophile Leukozyten. In diesem Falle wurde an Stelle von Bouillon nach dem oben geschilderten Spätschen Verfahren ca. $100 \mathrm{ccm}$ Wasserextrakt von Ascaris lumbricoides, das aus zerriebenen Würmern mit 10 fachem Volumen physiologischer Kochsalzlösung ausgezogen war, in die Bauchhöhle eines Kaninchens gebracht. Nach 24 Stunden wurde es wieder wie bei (1) aus der Bauchhöhle gespült. Man konnte unter dem Mikroskope erkennen, dass die auf diese Weise gewonnenen Leukozyten hauptsächlich Eosinophilen waren.

(d) Organe und Gewebe. Muskeln, Leber, Niere, Milz und Darm wurden einem aus der Karotis verbluteten Kaninchen entnommen, dessen sämtliche Organe dadurch blutfrei gemacht wurden, dass man kurz vor dem Tode des Tiers physiologische Kochsalzlösung durch die Aorta thoracalis hindurchströmen liess.

Tabelle

\begin{tabular}{|c|c|c|c|c|c|c|c|c|}
\hline Organe & $\stackrel{80}{\Xi}$ & 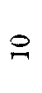 & B & 8 & $\stackrel{8}{\S}$ & ఠి & $\underset{\forall}{\stackrel{\gamma}{~}}$ & 8 \\
\hline Polynukleäre Leukozyten & 0,03 & + & + & + & + & + & + & + \\
\hline Eosinophile Leukozyten & $"$ & + & + & + & + & + & + & + \\
\hline Mononukleäre Leukozyten & , & + & + & + & + & + & + & + \\
\hline Polynukleüre Leukozyten & $"$ & + & + & + & + & + & + & + \\
\hline Muskel & $"$ & - & - & - & - & - & 一 & - \\
\hline Leber & $"$ & - & - & - & - & - & - & - \\
\hline Niere & $"$ & - & - & - & - & - & - & - \\
\hline Milz & $"$ & - & - & - & - & - & - & - \\
\hline Darn & $"$ & - & - & - & - & - & - & - \\
\hline Knochenmark & $"$ & + & \pm & - & - & - & - & - \\
\hline Lymphdrüse & $"$ & + & \pm & - & - & - & - & - \\
\hline Linse & $"$ & - & - & - & - & - & - & - \\
\hline Glaskörper & $"$ & - & - & - & - & - & - & - \\
\hline
\end{tabular}

Anmerkung: $\mathrm{N}=$ Stickstoffgehalt (mg \%) der Stammlösung (Antigen) 
(e) Andere Gewebe. Dem wie oben behandelten Tiere wurden Knochenmark, Lymphdrüse, Linse und Glaskörper als Versuchsmaterial entnommen.

Die Präzipitinreaktion wurde nach M. Ascolis Methode ausgeführt. Die Eiweisskonzentration der einzelnen Wasserextrakte wurde durch den Stickstoffgehalt bestimmt, der nach Kjelda hls Methode gemessen wurde. Das Versuchsresultat zeigt die folgende Tabelle.

Aus der Tabelle kann man erkennen, dass das polynukleäre Leukozytenimmunserum streng organspezifische Reaktion zeigt, weil es gegen das Wasserextrakt polynukleärer Leukozyten in 40 000-600 000 facher Verdünng stark positiv reagiert, während es gegen das Wasserextrakt mononukleïrer und eosinophiler Leukozyten kaum in 600 facher Verdünnung, gegen das von Knochenmark und Lymphdrüse in 10 facher Verdünnung reagiert, aber gegen das anderer Organe und Gewebe wie Muskel, Milz, Leber, Niere, Darm, Linse und Glaskörper gar keine Reaktion zeigt. Meines Erachtens müssen folglich die poly-, mononukleüren und eosinophilen Leukozyten ihre eigene immunologisch nachweisbare Organspezifität besitzen. Das polynukleäre Leukozytenimmunserum reagierte gegen die Wasserextrakte anderer Leukozytenarten positiv, wenn auch in geringerem Grade. Doch scheint das Antigen durch Beimengung etlicher andersartiger Leukozyten verunreinigt $z u$ sein, folglich wurde nur polyvalentes Immunserum geschaffen. Um ein streng monovalentes Immunserum zu bekommen, mit dem die einzelnen Leukozytenarten genau identifiziert wer-

I.

\begin{tabular}{|c|c|c|c|c|c|c|c|c|c|c|c|}
\hline$\underset{:}{\mathbb{E}}$ & $\overbrace{-1}^{8}$ & $\underset{8}{8}$ & $\underset{\forall}{8}$ & 8 & $\underbrace{8}_{\infty}$ & $\begin{array}{l}8 \\
\stackrel{1}{0} \\
0\end{array}$ & 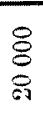 & 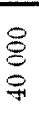 & $\begin{array}{l}8 \\
8 \\
8\end{array}$ & $\begin{array}{l}\stackrel{8}{8} \\
\stackrel{\Xi}{ }\end{array}$ & $\begin{array}{l}8 \\
8 \\
8 \\
\end{array}$ \\
\hline+ & + & 1 & + & + & + & t & + & + & + & 土 & - \\
\hline \pm & - & - & - & - & - & - & - & - & 1 & 亡 & - \\
\hline \pm & -- & - & - & - & - & - & - & - & - & - & - \\
\hline+ & + & + & + & + & + & + & + & + & tt & - & - \\
\hline$=$ & $=$ & $=$ & $=$ & $=$ & - & $=$ & 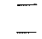 & $=$ & $I$ & $=$ & - \\
\hline- & - & - & - & - & - & - & - & - & - & - & - \\
\hline- & - & - & - & - & - & - & - & - & - & - & - \\
\hline - & - & - & - & - & - & - & - & - & - & - & $\rightarrow$ \\
\hline- & - & - & - & - & - & - & - & - & - & - & - \\
\hline- & - & - & - & - & - & - & - & - & - & $=$ & - \\
\hline$=$ & $\bar{z}$ & $=$ & - & $=$ & - & I & \pm & $\overline{-}$ & I & - & - \\
\hline
\end{tabular}


den könnten, müsste ganz reines Antigen geschaffen werden. Also möchte ich hieraus schliessen, dass die Leukozyten des Kaninchens höchstwahrscheinlich strenge Organspezifität besitzen.

\section{II. Über diespezifisch toxische Wirkung des Leukozytenimmunserums.}

Leschke und Sp $\ddot{a} \mathrm{t}^{13)}$ meinten auf Grund ihrer Experimente, dass es sich bei den nach Injektion von Leukozytenimmunserum auftretenden Symptomen um die Auflösung von Leukozyten handelt, ähnlich wie sich bei der Hämolyse Erythrozyten verhalten, während Maurine, P. et J. Galiaç ${ }^{14)}$ sie auf die Lenkozytenfragilität zurückführten. Um die toxische W irkung des polynukleären Leukotoxins festzustellen, injizierte ich das Serum einem Kaninchen intravenös, dessen Blutspiegel dann zu gewissen Fristen unter dem Mikroskope genau betrachtet wurde. Zuerst wurde zur Kontrolle der Blutspiegel eines Kaninchens verfolgt, dem etwas normales Hausentenserum intravenös eingeführt wurde und dann Hauptversuch an demselben Kaninchen ausgeführt. Das Resultat ist folgendes.

Tabelle II (1).

Kleine Dose.

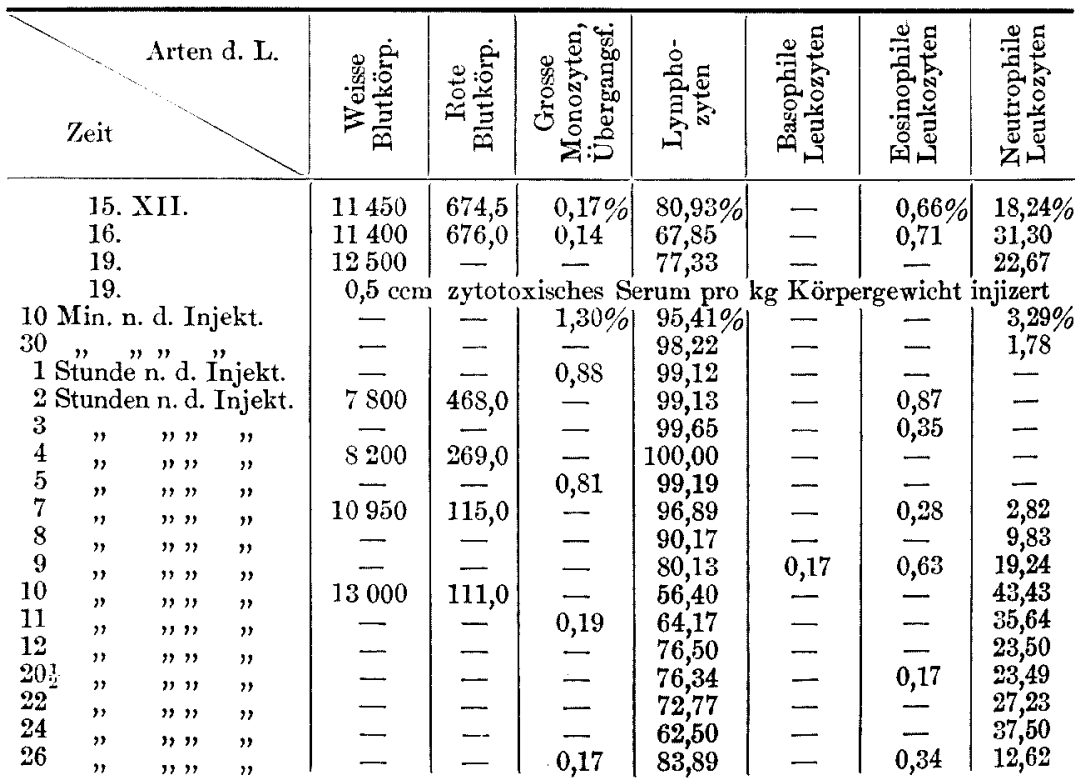

13) Spät, W. und Hoder, F., Zeitschr. f. Immunitätsf., 1927, 49, 382.

14) Maurine, P. et Galiacy, Cpt. Rend. Soc. Biol., 1923, 89, 1163. 
Tabelle II (2).

Kleine Dose (Kontrolle).

\begin{tabular}{|c|c|c|c|c|c|c|c|c|c|}
\hline \multicolumn{3}{|c|}{ Zeit } & 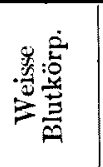 & 总 & 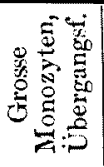 & 总 & 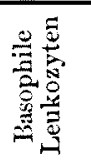 & 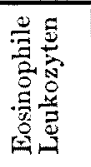 & 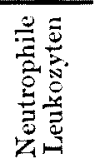 \\
\hline \multicolumn{3}{|c|}{ 16. I. } & 8150 & 712,5 & $\begin{array}{l}0,28 \% \\
0,45\end{array}$ & $\begin{array}{l}53,48 \% \\
33,23\end{array}$ & $\underline{0,28 \%}$ & $0,42 \%$ & $45,53 \%$ \\
\hline \multicolumn{3}{|c|}{$\begin{array}{l}17 . \\
17 .\end{array}$} & \multicolumn{7}{|c|}{$0,5 \mathrm{ccm}$ Normal-II Iausentenserum pro kg Körpergewicht in- } \\
\hline \multirow{3}{*}{\multicolumn{3}{|c|}{$\begin{array}{l}10 \text { Min. n. d. Injekt. } \\
30 \text { " ". " Mnjekt. } \\
1 \text { Stunde n. d. Injekt. } \\
2 \text { Stunden n. d. Injekt. }\end{array}$}} & $5 \$ 50$ & 696,0 & - & $75,00 \%$ & - & - & $25,00^{\circ} a$ \\
\hline & & & $\therefore 0$ & - & - & 67,65 & - & - & 32,35 \\
\hline & & & 3700 & - & & $\begin{array}{l}0 ., 19 \\
19,35\end{array}$ & z & $\begin{array}{l}0,40 \\
0,49\end{array}$ & $\begin{array}{l}81,91 \\
80,16\end{array}$ \\
\hline 3 & $"$ & $", "$ & 5750 & - & - & 30,47 & - & & 69,53 \\
\hline & $"$ & $" n$ & & & & 36,00 & - & 0,22 & 53,78 \\
\hline 5 & $"$ & $", "$, & 7400 & 738,5 & - & - & - & - & \\
\hline 6 & $"$ & $"$, & & - & 0,28 & 19,38 & - & - & 80,34 \\
\hline 7 & $"$ & $"$ & 7600 & - & & 31,84 & - & 0,45 & 67,72 \\
\hline 9 & $"$ & $" n$ & & - & 0,21 & 11,42 & - & 0,42 & 87,95 \\
\hline 11 & $"$ & ", , & - & 一 & & 15,50 & - & 0,26 & 84 \\
\hline 12 & $"$ & " " & - & - & 0,36 & 67,38 & - & 0,36 & 31,15 \\
\hline 21 & 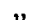 & , & & - & & 11,16 & & - & \\
\hline 27 &, & $"$ & 13080 & 743,5 & 0,31 & & & & 51,39 \\
\hline 35 & $"$ & $" n$ & & - & - & 57,89 & - & 0,24 & 41,87 \\
\hline
\end{tabular}

Tabelle II (3).

Mittlere Dose.

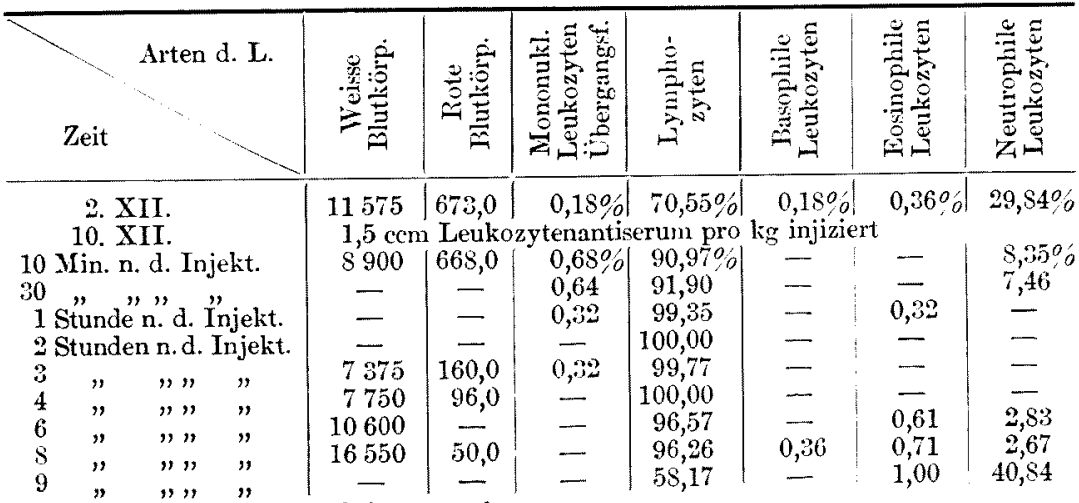

912 Stunden nach d. Injektion gestorben.

Tabelle II (4).

Mittlere Dose (Kontrolle)

\begin{tabular}{|c|c|c|c|c|c|c|c|}
\hline Arten d. L. & 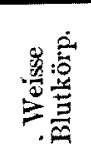 & 㝘 & 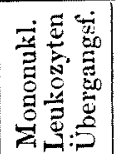 & 总咅 & 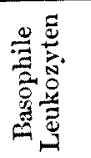 & 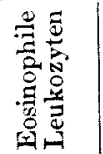 & 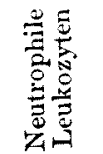 \\
\hline $\begin{array}{l}\text { 1. XII. XII. } \\
\text {. XII. }\end{array}$ & $\begin{array}{r}13300 \\
1,5 \mathrm{cc}\end{array}$ & $\begin{array}{l}638,7 \\
\text { Norm }\end{array}$ & $\begin{array}{r}0,13 \% \\
\text { I-Hausen }\end{array}$ & $\begin{array}{l}46,21 \% \\
\text { anserum1 }\end{array}$ & $\begin{array}{l}0,13 \% \\
0 \mathrm{~kg} \text { in }\end{array}$ & $\begin{array}{c}0,26 \% \\
\text { ziert }\end{array}$ & $53,27 \%$ \\
\hline
\end{tabular}




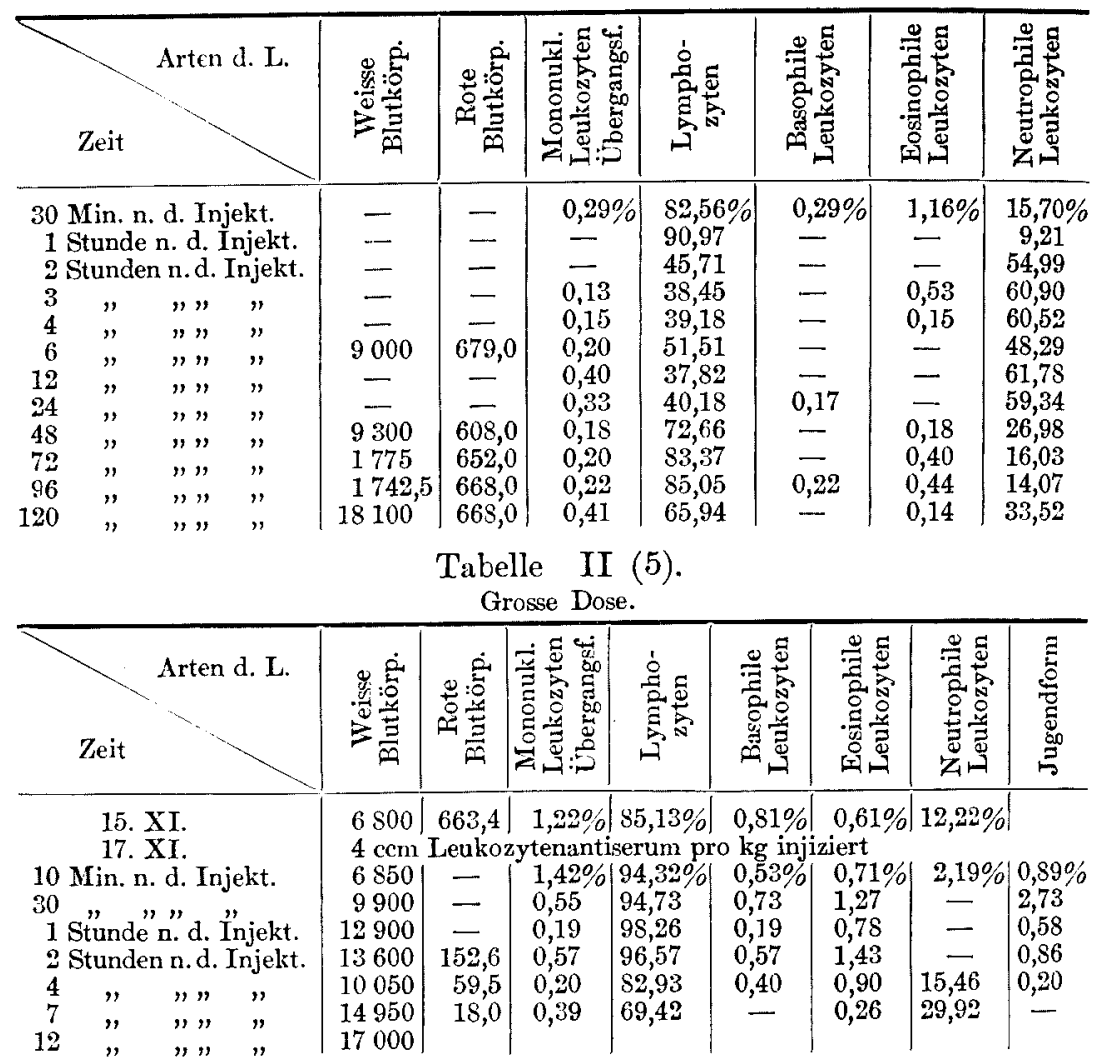

17 Stunden nach d. Injektion von toxischem Serum gestorben.

Tabelle II (6).

Grosse Dose (Kontrolle)

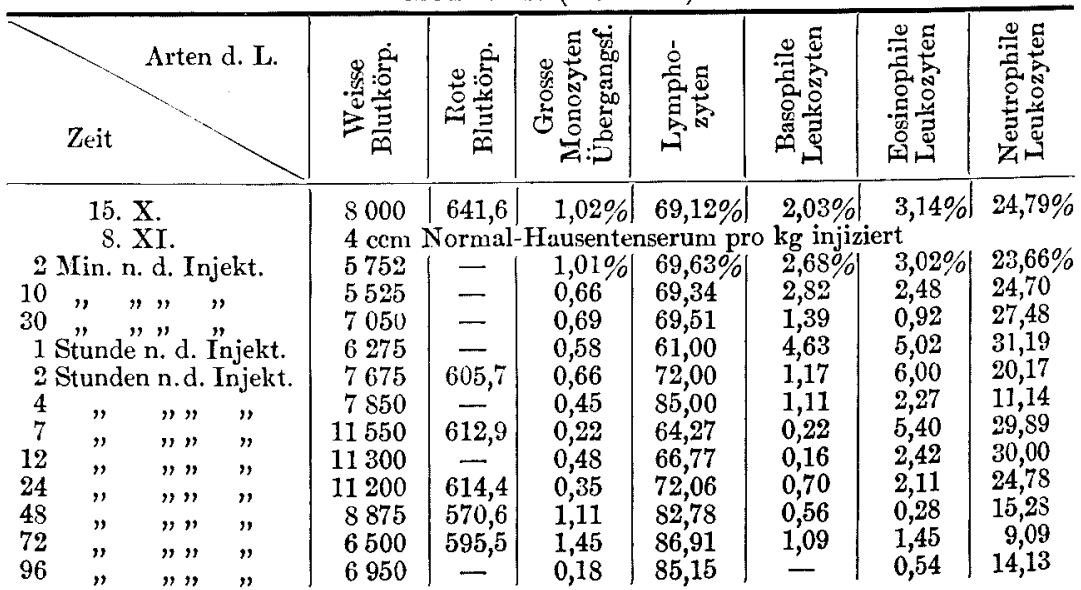


Fig. I.

Prozent der Lymphozyten und der polynukleären Leukozyten bei kleiner Dose.

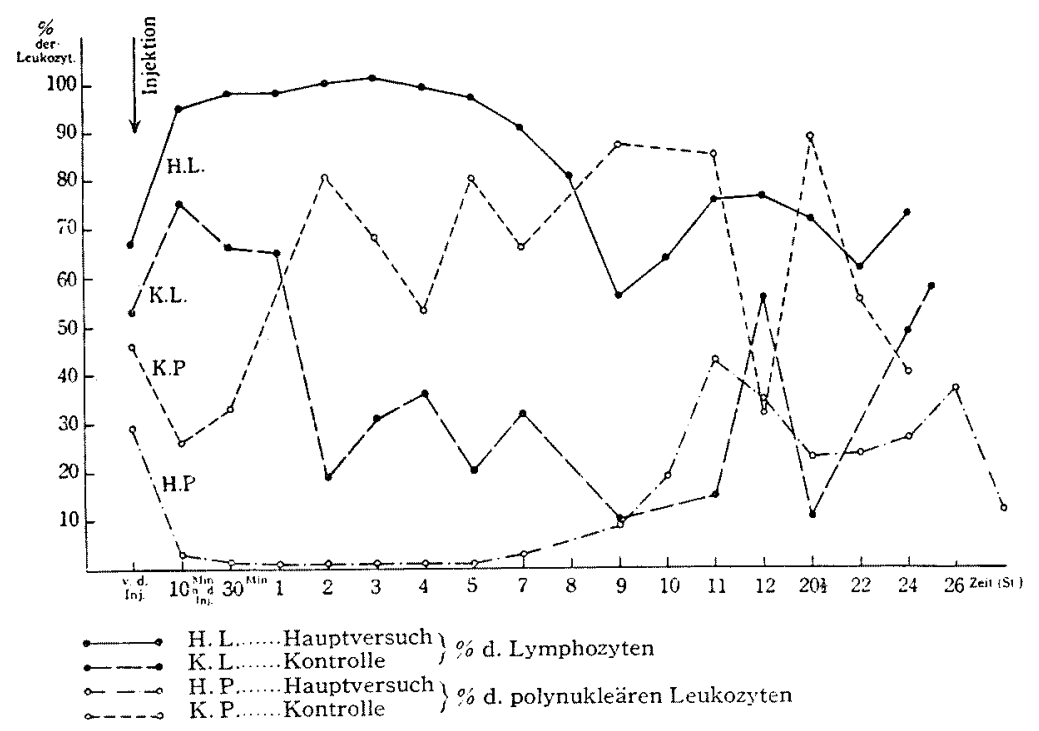

Fig. II.

Prozent der Lymphozyten und der polynukleären Leukozyten bei mittlerer Dose.

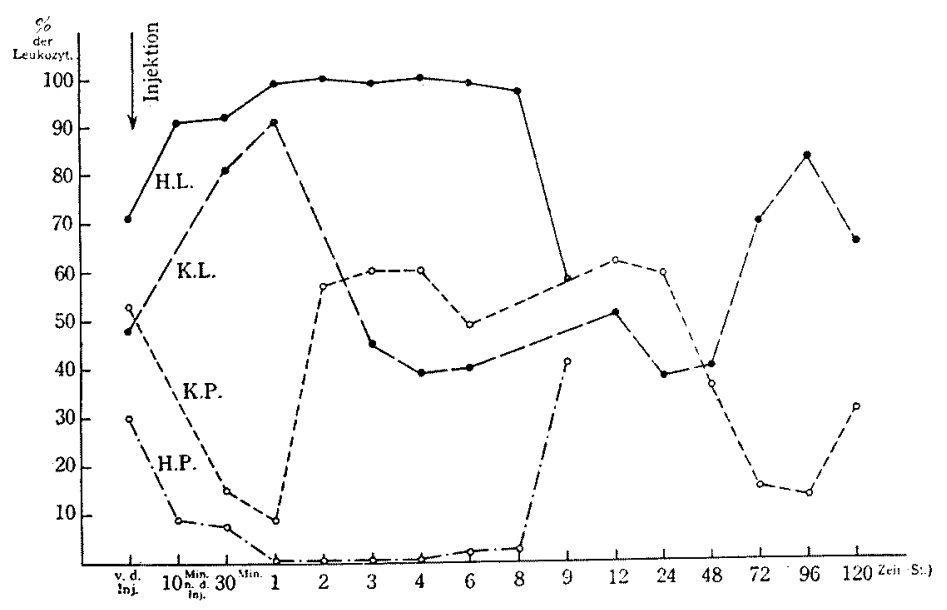


Fig. III.

Prozent der Lymphozyten und der polynukleären Leukozyten bei grosserer Dose.

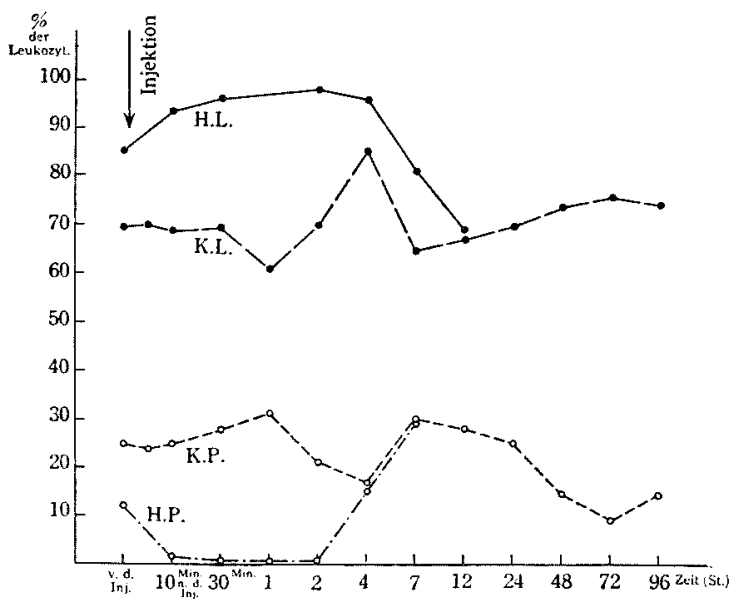

Wie obige Tabelle zeigt, können wir bei Injektion normalen Hausentenserums als Kontrolle allgemeineVermehrung der Leukozytenzahl erkennen, während das Verhältnis aller Leukozytenarten zueinander keine erhebliche Änderung zeigt. Injiziert man eine kleine Dose des genannten

Immunserums, so bietet der Blutspiegel nichts Besouderes dar, während bei einer mittleren oder grossen Menge die Versuchstiere in einigen Tagen zugrunde gehen. Eine Stunde nach der Injektion waren die polynukleären Leukozyten fast ganz aus der Blutbahn verschwunden, traten aber im Verlauf von 4 bis 7 Stunden wieder darin auf. Diese sehr interessante Erscheinung bedeutet eine elektive Einwirkung des Immunserums auf die polynukleären Leukozyten und ist ein Hinweis darauf anzusehen, das Leukotoxin eine ziemlich starke zytotoxische Eigenschaft besitzt.

\section{Zusammenfassung.}

1. Die Leukozyten des Kaninchens haben strenge Organspezifität, so dass sich jede Leukozytenart immunologisch identifizieren lässt.

2. Das polynukleäre Leukotoxin scheint auf die polynukleären Leukozyten eigentümlich schädigend zu wirken.

An dieser Stelle möchte ich Herrn Prof. 'T. Ishikawa, unter dessen Leitung die Ausf ührung der Versuche stattfand, meinen ergebensten Dank aussprechen. 